\title{
Minireview
}

\section{The role of the VEGF-CNEGFR-3 axis in cancer progression}

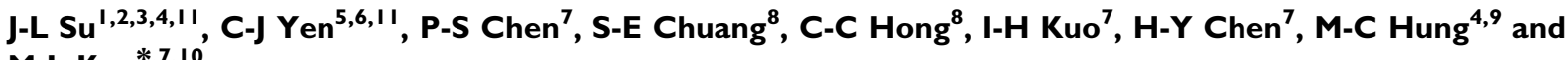 \\ M-L Kuo ${ }^{*, 7,10}$
}

'Institute of Medical Science, College of Medicine, China Medical University, Taichung 404, Taiwan; ${ }^{2}$ Center for Molecular Medical, China Medical University Hospital, Taichung 404, Taiwan; ${ }^{3}$ Department of Biotechnology and Bioinformatics, Asia University, Taichung 4I 354, Taiwan; ${ }^{4}$ Department of Molecular and Cellular Oncology, The University of Texas MD Anderson Cancer Center, Houston, TX 77030, USA; ${ }^{5}$ Department of Internal Medicine, National Cheng Kung University Hospital, Tainan 70428, Taiwan; '6nstitute of Clinical Medicine, National Cheng Kung University Hospital, Tainan 70428, Taiwan; ${ }^{7}$ Institute of Toxicology, College of Medicine, National Taiwan University, Taipei 100, Taiwan; ${ }^{8}$ Division of Cancer Research, National Health Research Institutes, Taipei 10016 , Taiwan; ${ }^{9}$ Graduate School of Biomedical Sciences, The University of Texas Health Science Center at Houston, Houston, TX 77030, USA; ${ }^{10}$ Angiogenesis Research Center, National Taiwan University, Taipei, Taiwan

Vascular endothelial growth factor (VEGF) receptor 3 (VEGFR-3) (also called VEGFR-3) is activated by its specific ligand, VEGF-C, which promotes cancer progression. The VEGF-CNEGFR-3 axis is expressed not only by lymphatic endothelial cells but also by a variety of human tumour cells. Activation of the VEGF-CNEGFR-3 axis in lymphatic endothelial cells can facilitate metastasis by increasing the formation of lymphatic vessels (lymphangiogenesis) within and around tumours. The VEGF-CNEGFR-3 axis plays a critical role in leukaemic cell proliferation, survival, and resistance to chemotherapy. Moreover, activation of the VEGF-C/VEGFR-3 axis in several types of solid tumours enhances cancer cell mobility and invasion capabilities, promoting cancer cell metastasis. In this review, we discuss the novel function and molecular mechanism of the VEGF-CNEGFR-3 axis in cancer progression.

British Journal of Cancer (2007) 96, 54I-545. doi:I0.1038/sj.bjc.6603487 www.bjcancer.com

Published online 12 December 2006

(c) 2007 Cancer Research UK

Keywords: VEGF-C; VEGFR-3; lymphangiogenesis; metastasis

Tumour invasion and metastasis are the critical steps in determining the aggressive phenotype of human cancers and the principal cause of cancer deaths. A better understanding of the molecular events that lead to metastasis and of the complex interactions between metastatic cells and host factors is essential for designing more effective cancer therapies. One of these events is the endogenous production of growth factors that act on tumours via functional external receptors.

The vascular endothelial growth factor (VEGF) gene family, which encodes five polypeptide growth factors, VEGF-A, -B, -C, -D, and $-E$, is particularly important because of its angiogenic and lymphangiogenic properties that promote the growth and metastasis of neoplasms (Ferrara and Davis-Smyth, 1997). Vascular endothelial growth factors achieve this by activating related tyrosine kinase receptors termed VEGFR-1 (Flt-1) and VEGFR-2 (KDR). These receptors are mainly expressed by endothelial cells (Ferrara and Davis-Smyth, 1997) but are also expressed by a wide variety of cancer cell lines (Liu et al, 1995; Strizzi et al, 2001; Wu et al, 2003). The expression of both VEGF receptors (VEGFRs) and their ligands, results in an autocrine function. For example, in

\footnotetext{
* Correspondence: Dr M-L Kuo, Laboratory of Molecular and Cellular Toxicology, Institute of Toxicology, College of Medicine, National Taiwan University, No. I, Section I, Jen-Ai Road, Taipei, Taiwan;

E-mail: toxkml@ha.mc.ntu.edu.tw

11 These authors contributed equally to this work.

Received 10 July 2006; revised 2 October 2006; accepted 25 October 2006; published online 12 December 2006
}

melanoma (Liu et al, 1995) and mesothelioma (Strizzi et al, 2001) cells, exogenous VEGF-A stimulates cell proliferation by activating VEGFR-2. Vascular endothelial growth factor-A also stimulates human leukaemic cell proliferation, migration, and matrix metalloproteinase 9 production through VEGFR-2 (Dias et al, 2002). In addition, VEGF-A induces the activation of mitogenactivated protein kinases (MAPKs), promotes cell growth in human pancreatic and breast cancer cells (von Marschall et al, 2000), and promotes cell adhesion and migration through activated integrin $\alpha \mathrm{v} \beta 3$ in M21 melanoma cells (Byzova et al, 2000). These reports highlight the importance of VEGFs/VEGFRs signaling in the biology of a variety of tumour cells and illustrate its well-described role.

Vascular endothelial growth factor- $\mathrm{C}$ has been characterised as a lymphangiogenic and angiogenic growth factor and has been shown to signal through the receptors VEGFR-3 (also called Flt-4) and VEGFR-2 (Plate, 2001). Vascular endothelial growth factor receptor 3 has also been shown to be important in determining the potential for a lymphangiogenic response. Recent studies have indicated that VEGFR-3, which has been proposed as a marker for lymphatic endothelial cells, is also expressed in a variety of human malignancies. Vascular endothelial growth factor receptor 3 expression in colon cancer has been associated with poorer survival, suggesting an axis between VEGF-C and VEGFR-3 in colorectal cancer (Witte et al, 2002). Expression of VEGFR-3 has also been reported to be significantly correlated with the different stages of cervical carcinogenesis (Van Trappen et al, 2003). The VEGF-C/VEGFR-3 axis exerts different biological effects on cancer cells to cause tumour progression. For example, in our previous 
study (Su et al, 2006), we provided evidence that the VEGF-C/ VEGFR-3 axis enhances cancer cell mobility and invasion capabilities and promotes cancer cell metastasis.

We concluded from these various observations that, the VEGFC/VEGFR-3 axis, through different signaling pathways, plays a critical role in cancer progression by regulating different cellular functions, such as invasion, proliferation, and resistance to chemotherapy.

\section{THE VEGF-C/VEGFR-3 AXIS AFFECTS TUMOUR PROGRESSION BY REGULATING LYMPHANGIOGENESIS}

Lymphangiogenesis and sustained angiogenesis are important steps in tumour progression. Similar to angiogenesis, a tumour can induce its own network of lymphatics that connect with the surrounding lymphatic vessels. However, clinical and pathological observations suggest that for many carcinomas, the transport of tumour cells by lymphatics is the most common pathway of initial dissemination, with cancer spread by afferent lymphatics following routes of natural drainage. Until recently, tumour-induced lymphangiogenesis was a relatively unfamiliar concept in the field of cancer research.

One could envisage that overproduction of lymphangiogenic factors may provide extra lymphatic channels, thus promoting the spread of tumour cells. It has been reported that two members of the VEGF family, VEGF-C and VEGF-D, not only are important regulators of lymphangiogenesis in vivo, but also enhance lymphatic metastasis (Karpanen and Alitalo, 2001; Skobe et al, 2001). Previous studies have demonstrated that VEGF-C is the lymphangiogenic factor that induces the formation of lymphatic vessels within and around tumours. In addition, these studies have shown that VEGF-C-overexpressing tumours increase intratumoral lymphangiogenesis by activating the VEGF-C/VEGFR-3 axis in lymphatic endothelial cells, enhancing metastatic spread via the lymphatics (Karpanen and Alitalo, 2001; Skobe et al, 2001). These discoveries affect how we currently think about tumours; that is, the spread of cancer cells from the primary tumour to the lymphatics and blood stream, which has been previously attributed to the general invasive properties of the tumour, is now understood to be due to active recruitment of new lymphatics by tumour-derived VEGF-C or VEGF-D (Karpanen and Alitalo, 2001; Plate, 2001; Karkkainen et al, 2002).

In animal models, the induction of lymphangiogenesis by the VEGF-C/VEGFR-3 axis increased tumour metastasis via the lymphatic system (Plate, 2001; Skobe et al, 2001). Vascular endothelial growth factor- $\mathrm{C}$ has been detected in many human cancers, and a number of reports have shown a correlation between VEGF-C expression in human tumours and the formation of metastases in regional lymph nodes. So far, the VEGF-C levels in several types of primary tumours have correlated significantly with lymph node metastasis (Akagi et al, 2000; Hashimoto et al, 2001; Witte et al, 2002; Juttner et al, 2006; Su et al, 2006). However, there are also some reports did not find a correlation between VEGF-C expression and metastasis (Gunningham et al, 2000; George et al, 2001; Komuro et al, 2001; Hanrahan et al, 2003; Koyama et al, 2003).

VEGF-C overexpressing tumours not only increase intratumoral lymphangiogenesis but also the peri-tumoral amount of lymphatic vessels. There are several papers report that peri-tumoral, but not intratumoral, lymphatic vessels were shown to be functional and it was propose that lymphatic vessels at the tumour margin may play a major role in lymphatic spread of tumour cells (Padera et al, 2002; Gombos et al, 2005). An important point in the field of lymphangiogenesis is to find out whether intratumour lymphatic vessels are functional for tumour dissemination into the lymph node.

\section{THE ROLE OF THE VEGF-C/VEGFR-3 AXIS IN TUMOUR CELLS}

Vascular endothelial growth factor receptors are primarily expressed by endothelial cells, but are also expressed by nonendothelial cell types, including several cancer cells (Weninger et al, 1999; Yonemura et al, 2001; Witte et al, 2002; Li et al, 2003; Neuchrist et al, 2003; Van Trappen et al, 2003; Wu et al, 2003). Vascular endothelial growth factors play a critical role in pathological conditions by binding to and activating VEGFRs. Many tumour cells and tumour-associated vasculature express both VEGFRs and VEGFs, and an autocrine/paracrine function has been proposed (Byzova et al, 2000; Dias et al, 2002).

Although expression of VEGF-C and VEGFR-3 has been significantly and negatively correlated to the progression of certain selective types of cancer (Table 1), the function of the VEGF-C/ VEGFR-3 axis in cancer cells is largely unknown. Vascular endothelial growth factor receptor 3 expression in colon cancer, which as noted earlier has been associated with poorer survival (Witte et al, 2002) and has been significantly correlated with the different stages of cervical carcinogenesis (Van Trappen et al, 2003), suggesting an axis between VEGF-C and VEGFR-3 in cancer cells. However, there are some studies that did not detect VEGFR-3 expression by tumour cells (Chen et al, 2004; Zeng et al, 2004) and these controversial results might be clarified by more powerful and sensitive technique in the future.

Immunohistochemical staining has shown that VEGFR-3 is expressed by Kaposi sarcoma cells (Weninger et al, 1999). Furthermore, Marchio et al (1999) reported that tyrosine phosphorylation of VEGFR-3 was increased in Kaposi sarcoma cells treated with VEGF-C recombinant protein as well as C156S mutant VEGF-C recombinant protein, a selective ligand and an activator of VEGFR-3, although devoid of any Flt-1 activation property. Marchio et al (1999) found that the activation of the VEGF-C/VEGFR-3 axis in Kaposi sarcoma cells was indeed involved in the regulation of cellular functions, such as proliferation and migration. Using endothelial cells as a control, Marchio et al (1999) further found that the activation of the VEGF-C/ VEGFR-3 axis significantly increased the proliferation and migration of KS IMM Kaposi sarcoma cells in a dose-dependent manner.

The VEGF-C/VEGFR-3 axis has also been found to play a role in the growth of malignant mesothelioma cells (Masood et al, 2003). Indeed, both the VEGF-A/VEGFR-2 and VEGF-C/VEGFR-3 axes are present in several malignant pleural mesothelioma cell lines, and both axes are functionally related to cell growth. Further supporting the role of the VEGF-C/VEGFR-3 axis in cell growth was the finding that using VEGF-C antisense oligonucleotides, recombinant VEGFR-3/Fc, or VEGFR-3 antibody to inhibit the activity of the VEGF-C/VEGFR-3 axis in malignant pleural mesothelioma cells resulted in a significant reduction in cell viability. However, there are now many studies, including our previous study, demonstrate that VEGF-C overexpression by experimental tumours did not enhance tumour growth but selectively promote tumour metastasis (Skobe et al, 2001; Hoshida et al, 2006; Su et al, 2006). In addition, other studies have been reported that neither expression of VEGFR-3-Ig in LNM35 lung cancer cells (He et al, 2002) nor expression of VEGF-C-specific small interfering RNA vector in C166 mammary tumour cells (Chen et al, 2005) changed the tumour growth rate.

The interaction of VEGF-C with VEGFR-3 in leukaemia cells promotes cell survival and proliferation, as shown by Dias et al (2002) in two cell lines and in five cases of VEGFR- $3^{+}$primary leukaemia. In particular, these researchers observed that VEGF-C and a mutant form of the molecule that lacks the KDR-binding motif induced receptor phosphorylation and increased cell proliferation and survival, as shown by increased Bcl-2/Bax ratios. Moreover, the activation of the VEGF-C/VEGFR-3 axis protected 
Role of the VEGF-C/VEGFR-3 axis during cancer progression

Table I Expression, function, and clinical significance of VEGFR-3 in human tumours

\begin{tabular}{ll}
\hline Tumour type & Primary findings \\
\hline Gastric cancer & $\begin{array}{l}\text { The expression of VEGFR-3 correlated with reduced carcinoma-specific survival, and a Cox multivariate } \\
\text { regression analysis qualified VEGFR-3 as an independent prognostic parameter. The presence of the } \\
\text { VEGF-CNEGFR-3 axis was associated with poor survival in lymph node-positive gastric cancer }\end{array}$ \\
& The expression of VEGFR-3 in breast cancer tissue was not significantly related to tumour grade $(P=0.063)$ \\
& The activation of the VEGF-CNEGFR-3 axis increased cell mobility of breast cancer cells. The expression of \\
& VEGFR-3 in breast tumour tissue was higher than it was in matched normal tissue \\
& VEGF-C and VEGFR-3 status may be indicative of survival rates for patients with TI lung adenocarcinoma \\
& VEGF-C and VEGFR-3 expression may be indicative of survival rates for patients with NSCLC \\
& In NSCLC, the VEGF-CNEGFR-3 axis is related to lymphangiogenesis, and angiogenesis and to the occurrence \\
& and development of lung cancers. VEGF-C expression could be a useful predictor of poor prognosis in NSCLC \\
& The VEGF-CNEGFR-3 axis enhances cancer cell mobility and invasiveness and contributes to the promotion of \\
& cancer cell metastasis Examination of tumour tissues from various types of cancers revealed high levels of \\
& VEGFR-3 and VEGF-C expression that correlated closely with clinical metastasis and patient survival
\end{tabular}

\section{References}

Juttner et al (2006)

Longatto Filho et al (2005)

Su et al (2006)

Kojima et al (2005)

Arinaga et al (2003)

Li et al (2003)

Su et al (2006)

Dias et al (2002) VEGF-CNEGFR-3 pathway is a novel therapeutic target for the treatment of subsets of acute leukaemia

Cervical cancer

A significant positive correlation was found between VEGF-C and VEGFR-3 expression through the different stages of cervical carcinogenesis. These findings suggest an autocrine growth stimulation pattern via VEGFR-3 in cervical carcinoma cells

Colorectal

cancer

The expression of VEGFR-3 in $>25 \%$ of the cancer cells was associated with significantly poorer overall survival $(P<0.05)$, but not with lymph node metastasis or depth of tumour invasion These results suggest that VEGFs promote cancer growth not only by stimulating angiogenesis, but also by acting on receptors present on the cancer cells themselves

The expression of VEGFR-3 in colorectal tumour tissue was higher than it was in matched normal tissue

Prostate cancer

Significantly upregulated expressions of VEGF-A, VEGF-C, and VEGFR-3 were all found in malignant epithelium/ cancer cells compared with adjacent benign epithelium $(P<0.0 \mathrm{I})$ Patients in stage $D$ had a significantly higher score than did patients in stage $A, B$, or $C$ when comparing the expression of VEGF-C or VEGFR-3 in the tumour area $(P<0.0 \mathrm{I})$

The increased expression of the VEGF-C NEGFR-3 axis played a role in prostate cancer progression and in metastasis to regional lymph nodes

VEGFR-3 expression was associated with tumour progression and may play an important role in facilitating the lymphatic spread of prostate carcinomas; a high level of VEGFR-3 in prostate cancer cells increases the risk of biochemical recurrence in prostate cancer patients treated by radical prostatectomy

Kaposi sarcoma

The VEGF-CNEGFR-3 axis stimulates the migration and proliferation of Kaposi sarcoma cells

Marchio et al (1999)

The broad expression of the VEGF-CNEGFR-3 axis in head and neck squamous cell carcinoma suggests involvement in tumour lymphangiogenesis and angiogenesis, promoting tumour growth, and propagation of cancer cells. This implies that inhibitors of lymphangiogenesis could become effective therapeutic options

squamous cell

carcinoma

The presence of VEGF-D and VEGFR-3 in endometrial carcinoma may predict myometrial invasion and lymph node metastasis and may prospectively identify patients who are at increased risk for poor outcome. In addition, VEGF-D and VEGFR-3 may be promising targets for new therapeutic strategies in endometrial carcinoma
Van Trappen et al (2003)

Witte et al (2002)

Yang et al (2006)

Jennbacken et al (2005)

Li et al (2004)

Neuchrist et al (2003)

Yokoyama et al (2003)

Masood et al (2003)
Su et al (2006)

$\mathrm{NSCLC}=$ non-small cell lung cancer; VEGF $=$ vascular endothelial growth factor; VEGFR $=$ VEGF receptor.

leukaemic cells from the apoptotic effects of chemotherapeutic agents such as cytarabine, daunorubicin, and etoposide. These results also identified the VEGF-C/VEGFR-3 pathway as a novel therapeutic target for the treatment of subsets of acute leukaemia.

Our recent study ( $\mathrm{Su}$ et al, 2006) provided evidence that the VEGF-C/VEGFR-3 axis enhances cancer cell mobility and invasiveness and contributes to the promotion of cancer cell metastasis in various types of cancer, including lung adenocarcinoma, breast cancer, cervical prostate cancer, and colorectal cancer. Further, the VEGF-C/VEGFR-3-mediated invasion and metastasis of cancer cells was found to require upregulation of the neural cell adhesion molecule contactin-1 through activation of the Src-p38 MAPK-C/ EBP-dependent pathway. In agreement with the role of the
VEGF-C/VEGFR-3 axis in invasion and metastasis, an examination of tumour tissues from various types of cancers revealed high levels of VEGFR-3 and VEGF-C expression that correlated closely with clinical metastasis and patient survival. The function and molecular mechanism of the VEGF-C/VEGFR-3 axis were revealed from in vitro and in vivo studies and in an examination of patient outcomes.

\section{CONCLUSIONS}

The VEGF-C/VEGFR-3 axis plays a critical role in cancer progression by inducing lymphangiogenesis and facilitating the 
mobility of several types of cancer cells (Su et al, 2006). The VEGFC/VEGFR-3 axis may affect cancer development or progression by directly affecting tumour cells. Unlike the well-characterised axis of VEGF-A and VEGFR-2, there may be many undefined functions and molecular mechanisms involved in the tumour progression mediated by the VEGF-C/VEGFR-3 axis; thus, further study of the axis is needed. It is also important to identify the functions of the VEGF-C/VEGFR-3 axis in macrophage infiltration/activation and to determine how these tumour-educated macrophages affect tumour progression. This is because infiltrating macrophages have correlated significantly and negatively with cancer patient prognosis and survival (Ogawa et al, 2004; Schoppmann et al, 2006). It has been reported that tumour-associated macrophages play a crucial role in angiogenesis and lymphangiogenesis under inflammatory condition (Cursiefen et al, 2004; Maruyama et al, 2005). It also has been reported that macrophages support lymphangiogenesis in two different ways, either by transdifferentiating and directly incorporating into the endothelial layer or by stimulating division of pre-existent local lymphatic endothelial cells (Kerjaschki, 2005; Maruyama et al, 2005). In our unpublished data, we also found that VEGF-C plays a critical role in the macrophage infiltration in lung tumour through VEGFR-3 and this VEGFR-3-mediated macrophage infiltration may involved in radiosensitisation of lung cancer cells.

Another important area to be further explored is the nuclear localisation of VEGFR-3 and other VEGF receptors. This is because many receptor tyrosine kinases are detected in the cell nucleus and function as transcription cofactors to activate gene promoters, such as the EGFR family (Lo and Hung, 2006). Nuclear translocation of EGFRs has been reported to play a critical role in cancer progression (Lo and Hung, 2006). We have found that
VEGF-C activated VEGFR-3 was translocated into the nucleus of both lung adenocarcinoma cells and primary lymphatic endothelial cells (our unpublished data). Other aspects of the physical functions and molecular mechanisms of this nuclear localisation remain unclear and need to be clarified.

Targeting the VEGF-C/VEGFR-3 axis may be therapeutically significant for certain types of tumours. Thus, the continued discovery and characterisation of factors that regulate VEGF-C or VEGFR-3 will be essential for developing new therapies that limit the spread of cancer. In particular, new drugs that block the VEGFC/VEGFR-3 signaling pathway may provide useful anticancer therapeutics by mechanisms other than the blockage of lymphangiogenesis. In addition to being potential targets for inhibiting tumour metastasis, factors implicated in tumour lymphangiogenesis and the specific molecules found on the activated lymphatic endothelium may prove valuable in the diagnosis of particularly aggressive metastatic cancers.

\section{ACKNOWLEDGEMENTS}

This work was supported by National Science Council, Taiwan, Grants NSC93-2323-B-002-007, NSC93-2320-B-002-019, and NSC9323230-B-002-047; Taiwan Merit Scholarships TMS-094-2-B-023; Ministry of Economic Affairs, Taipei, Taiwan, Grant 93-EC17-A-19-S1-0016; National Taiwan University Hospital Grant NTUH-93A15-3. This work was supported in part by the Odyssey Program and The Cockrell Foundation Award for Scientific Achievement at The University of Texas MD Anderson Cancer Center. The authors have declared that no conflict of interest exists.

\section{REFERENCES}

Akagi K, Ikeda Y, Miyazaki M, Abe T, Kinoshita J, Maehara Y, Sugimachi K (2000) Vascular endothelial growth factor-C (VEGF-C) expression in human colorectal cancer tissues. Br J Cancer 83: 887-891

Arinaga M, Noguchi T, Takeno S, Chujo M, Miura T, Uchida Y (2003) Clinical significance of vascular endothelial growth factor $C$ and vascular endothelial growth factor receptor 3 in patients with nonsmall cell lung carcinoma. Cancer 97: 457-464

Byzova TV, Goldman CK, Pampori N, Thomas KA, Bett A, Shattil SJ, Plow EF (2000) A mechanism for modulation of cellular responses to VEGF: activation of the integrins. Mol Cell 6: 851-860

Chen F, Takenaka K, Ogawa E, Yanagihara K, Otake Y, Wada H, Tanaka F (2004) Flt-4-positive endothelial cell density and its clinical significance in non-small cell lung cancer. Clin Cancer Res 10: 8548-8553

Chen Z, Varney ML, Backora MW, Cowan K, Solheim JC, Talmadge JE, Singh RK (2005) Down-regulation of vascular endothelial cell growth factor-C expression using small interfering RNA vectors in mammary tumors inhibits tumor lymphangiogenesis and spontaneous metastasis and enhances survival. Cancer Res 65: 9004-9011

Cursiefen C, Chen L, Borges LP, Jackson D, Cao J, Radziejewski C, D’Amore PA, Dana MR, Wiegand SJ, Streilein JW (2004) VEGF-A stimulates lymphangiogenesis and hemangiogenesis in inflammatory neovascularization via macrophage recruitment. J Clin Invest 113: 1040-1050

Dias S, Choy M, Alitalo K, Rafii S (2002) Vascular endothelial growth factor (VEGF)-C signaling through VEGFR-3 (VEGFR-3) mediates leukemic cell proliferation, survival, and resistance to chemotherapy. Blood 99: 2179-2184

Ferrara N, Davis-Smyth T (1997) The biology of vascular endothelial growth factor. Endocr Rev 18: 4-25

George ML, Tutton MG, Janssen F, Arnaout A, Abulafi AM, Eccles SA, Swift RI (2001) VEGF-A, VEGF-C, and VEGF-D in colorectal cancer progression. Neoplasia 3: 420-427

Gombos Z, Xu X, Chu CS, Zhang PJ, Acs G (2005) Peritumoral lymphatic vessel density and vascular endothelial growth factor $C$ expression in early-stage squamous cell carcinoma of the uterine cervix. Clin Cancer Res 11: $8364-8371$
Gunningham SP, Currie MJ, Han C, Robinson BA, Scott PA, Harris AL, Fox SB (2000) The short form of the alternatively spliced flt-4 but not its ligand vascular endothelial growth factor $\mathrm{C}$ is related to lymph node metastasis in human breast cancers. Clin Cancer Res 6: $4278-4286$

Hanrahan V, Currie MJ, Gunningham SP, Morrin HR, Scott PA, Robinson BA, Fox SB (2003) The angiogenic switch for vascular endothelial growth factor (VEGF)-A, VEGF-B, VEGF-C, and VEGF-D in the adenomacarcinoma sequence during colorectal cancer progression. I Pathol 200: $183-194$

Hashimoto I, Kodama J, Seki N, Hongo A, Yoshinouchi M, Okuda H, Kudo $\mathrm{T}$ (2001) Vascular endothelial growth factor-C expression and its relationship to pelvic lymph node status in invasive cervical cancer. $\mathrm{Br}$ J Cancer 85: 93-97

He Y, Kozaki K, Karpanen T, Koshikawa K, Yla-Herttuala S, Takahashi T, Alitalo K (2002) Suppression of tumor lymphangiogenesis and lymph node metastasis by blocking vascular endothelial growth factor receptor 3 signaling. J Natl Cancer Inst 94: 819-825

Hoshida T, Isaka N, Hagendoorn J, di Tomaso E, Chen YL, Pytowski B, Fukumura D, Padera TP, Jain RK (2006) Imaging steps of lymphatic metastasis reveals that vascular endothelial growth factor- $\mathrm{C}$ increases metastasis by increasing delivery of cancer cells to lymph nodes: therapeutic implications. Cancer Res 66: 8065-8075

Jennbacken K, Vallbo C, Wang W, Damber JE (2005) Expression of vascular endothelial growth factor C (VEGF-C) and VEGF receptor-3 in human prostate cancer is associated with regional lymph node metastasis. Prostate 65: $110-116$

Juttner S, Wissmann C, Jons T, Vieth M, Hertel J, Gretschel S, Schlag PM, Kemmner W, Hocker M (2006) Vascular endothelial growth factor-D and its receptor VEGFR-3: two novel independent prognostic markers in gastric adenocarcinoma. J Clin Oncol 24: 228-240

Karkkainen MJ, Makinen T, Alitalo K (2002) Lymphatic endothelium: a new frontier of metastasis research. Nat Cell Biol 4: E2-E5

Karpanen T, Alitalo K (2001) Lymphatic vessels as targets of tumor therapy? J Exp Med 194: F37-F42 
Kerjaschki D (2005) The crucial role of macrophages in lymphangiogenesis. J Clin Invest 115: 2316-2319

Kojima H, Shijubo N, Yamada G, Ichimiya S, Abe S, Satoh M, Sato N (2005) Clinical significance of vascular endothelial growth factor-C and vascular endothelial growth factor receptor 3 in patients with T1 lung adenocarcinoma. Cancer 104: $1668-1677$

Komuro H, Kaneko S, Kaneko M, Nakanishi Y (2001) Expression of angiogenic factors and tumor progression in human neuroblastoma. J Cancer Res Clin Oncol 127: 739-743

Koyama Y, Kaneko K, Akazawa K, Kanbayashi C, Kanda T, Hatakeyama K (2003) Vascular endothelial growth factor-C and vascular endothelial growth factor-d messenger RNA expression in breast cancer: association with lymph node metastasis. Clin Breast Cancer 4: 354-360

Li Q, Dong X, Gu W, Qiu X, Wang E (2003) Clinical significance of coexpression of VEGF-C and VEGFR-3 in non-small cell lung cancer. Chin Med J (England) 116: 727-730

Li R, Younes M, Wheeler TM, Scardino P, Ohori M, Frolov A, Ayala G (2004) Expression of vascular endothelial growth factor receptor-3 (VEGFR-3) in human prostate. Prostate 58: 193-199

Liu B, Earl HM, Baban D, Shoaibi M, Fabra A, Kerr DJ, Seymour LW (1995) Melanoma cell lines express VEGF receptor KDR and respond to exogenously added VEGF. Biochem Biophys Res Commun 217: 721-727

Lo HW, Hung MC (2006) Nuclear EGFR signalling network in cancers: linking EGFR pathway to cell cycle progression, nitric oxide pathway and patient survival. $\mathrm{Br} J$ Cancer 94: 184- 188

Longatto Filho A, Martins A, Costa SM, Schmitt FC (2005) VEGFR-3 expression in breast cancer tissue is not restricted to lymphatic vessels. Pathol Res Pract 201: 93 - 99

Marchio S, Primo L, Pagano M, Palestro G, Albini A, Veikkola T, Cascone I, Alitalo K, Bussolino F (1999) Vascular endothelial growth factor-C stimulates the migration and proliferation of Kaposi's sarcoma cells. J Biol Chem 274: 27617-27622

Maruyama K, Ii M, Cursiefen C, Jackson DG, Keino H, Tomita M, Van Rooijen N, Takenaka H, D'Amore PA, Stein-Streilein J, Losordo DW, Streilein JW (2005) Inflammation-induced lymphangiogenesis in the cornea arises from CD11b-positive macrophages. J Clin Invest 115: $2363-2372$

Masood R, Kundra A, Zhu S, Xia G, Scalia P, Smith DL, Gill PS (2003) Malignant mesothelioma growth inhibition by agents that target the VEGF and VEGF-C autocrine loops. Int J Cancer 104: 603-610

Neuchrist C, Erovic BM, Handisurya A, Fischer MB, Steiner GE, Hollemann D, Gedlicka C, Saaristo A, Burian M (2003) Vascular endothelial growth factor $\mathrm{C}$ and vascular endothelial growth factor receptor 3 expression in squamous cell carcinomas of the head and neck. Head Neck 25: 464-474

Ogawa E, Takenaka K, Yanagihara K, Kurozumi M, Manabe T, Wada H, Tanaka F (2004) Clinical significance of VEGF-C status in tumour cells and stromal macrophages in non-small cell lung cancer patients. $\mathrm{Br} J$ Cancer 91: 498-503

Padera TP, Kadambi A, di Tomaso E, Carreira CM, Brown EB, Boucher Y, Choi NC, Mathisen D, Wain J, Mark EJ, Munn LL, Jain RK (2002) Lymphatic metastasis in the absence of functional intratumor lymphatics. Science 296: $1883-1886$

Plate K (2001) From angiogenesis to lymphangiogenesis. Nat Med 7: 151-152

Schoppmann SF, Fenzl A, Nagy K, Unger S, Bayer G, Geleff S, Gnant M, Horvat R, Jakesz R, Birner P (2006) VEGF-C expressing tumor-associated macrophages in lymph node positive breast cancer: impact on lymphangiogenesis and survival. Surgery 139: 839-846

Skobe M, Hawighorst T, Jackson DG, Prevo R, Janes L, Velasco P, Riccardi L, Alitalo K, Claffey K, Detmar M (2001) Induction of tumor lymphangiogenesis by VEGF-C promotes breast cancer metastasis. Nat Med 7: $192-198$

Strizzi L, Catalano A, Vianale G, Orecchia S, Casalini A, Tassi G, Puntoni R, Mutti L, Procopio A (2001) Vascular endothelial growth factor is an autocrine growth factor in human malignant mesothelioma. J Pathol 193: $468-475$

Su JL, Yang PC, Shih JY, Yang CY, Wei LH, Hsieh CY, Chou CH, Jeng YM, Wang MY, Chang KJ, Hung MC, Kuo ML (2006) The VEGF-C/VEGFR-3 axis promotes invasion and metastasis of cancer cells. Cancer Cell 9: $209-223$

Van Trappen PO, Steele D, Lowe DG, Baithun S, Beasley N, Thiele W, Weich H, Krishnan J, Shepherd JH, Pepper MS, Jackson DG, Sleeman JP, Jacobs IJ (2003) Expression of vascular endothelial growth factor (VEGF)-C and VEGF-D, and their receptor VEGFR-3, during different stages of cervical carcinogenesis. J Pathol 201: 544-554

von Marschall Z, Cramer T, Hocker M, Burde R, Plath T, Schirner M, Heidenreich R, Breier G, Riecken EO, Wiedenmann B, Rosewicz S (2000) De novo expression of vascular endothelial growth factor in human pancreatic cancer: evidence for an autocrine mitogenic loop. Gastroenterology 119: $1358-1372$

Weninger W, Partanen TA, Breiteneder-Geleff S, Mayer C, Kowalski H, Mildner M, Pammer J, Sturzl M, Kerjaschki D, Alitalo K, Tschachler E (1999) Expression of vascular endothelial growth factor receptor-3 and podoplanin suggests a lymphatic endothelial cell origin of Kaposi's sarcoma tumor cells. Lab Invest 79: 243-251

Witte D, Thomas A, Ali N, Carlson N, Younes M (2002) Expression of the vascular endothelial growth factor receptor-3 (VEGFR-3) and its ligand VEGF-C in human colorectal adenocarcinoma. Anticancer Res 22: $1463-1466$

Wu W, Shu X, Hovsepyan H, Mosteller RD, Broek D (2003) VEGF receptor expression and signaling in human bladder tumors. Oncogene 22: $3361-3370$

Yang J, Wu HF, Qian LX, Zhang W, Hua LX, Yu ML, Wang Z, Xu ZQ, Sui YG, Wang XR (2006) Increased expressions of vascular endothelial growth factor (VEGF), VEGF-C and VEGF receptor-3 in prostate cancer tissue are associated with tumor progression. Asian J Androl 8: $169-175$

Yokoyama Y, Charnock-Jones DS, Licence D, Yanaihara A, Hastings JM, Holland CM, Emoto M, Sakamoto A, Sakamoto T, Maruyama H, Sato S, Mizunuma H, Smith SK (2003) Expression of vascular endothelial growth factor (VEGF)-D and its receptor, VEGF receptor 3, as a prognostic factor in endometrial carcinoma. Clin Cancer Res 9: 1361-1369

Yonemura Y, Fushida S, Bando E, Kinoshita K, Miwa K, Endo Y, Sugiyama K, Partanen T, Yamamoto H, Sasaki T (2001) Lymphangiogenesis and the vascular endothelial growth factor receptor (VEGFR)-3 in gastric cancer. Eur J Cancer 37: 918-923

Zeng Y, Opeskin K, Baldwin ME, Horvath LG, Achen MG, Stacker SA, Sutherland RL, Williams ED (2004) Expression of vascular endothelial growth factor receptor-3 by lymphatic endothelial cells is associated with lymph node metastasis in prostate cancer. Clin Cancer Res 10: $5137-5144$ 